\title{
Evaluación del grado de sensibilización en el acero inoxidable AISI 304
}

\author{
O. González* y G. Santos*
}

Resumen El acero inoxidable austenítico, cuando se calienta en un rango de temperatura entre 550 y $850^{\circ} \mathrm{C}$ es susceptible a corrosión intergranular en ácidos. Este fenómeno, conocido como sensibilización, es resultado de la precipitación de carburos de cromo en el límite de grano, haciendo esas áreas menos resistentes a la corrosión. Se comparan dos pruebas diferentes de reactivación electroquímica con una prueba destructiva, relacionándolas con su respectiva microestructura. Se estableció una metodología cuantitativa para evaluar el grado de sensibilización del acero AISI 304 y se comparó con los datos generados de una herramienta no destructiva de campo, automatica y portatil, para medir el grado de sensibilización. Las técnicas electroquímicas usadas fueron: la EPR (Reactivación electroquímica Potenciocinética) o la prueba de curva simple, y la PRP (Reactivación Potenciocinética de Pasivación) o prueba de curva doble. La prueba destructiva usada fue la de sulfato férrico ( $50 \%$ ácido sulfúrico en ebullición durante 120 h, de acuerdo a la práctica estándar ASTM A-262, práctica B). La clasificación de la estructura se realizó de acuerdo a la práctica estándar ASTM A-262 A.

Palabras clave Sensibilización. Acero inoxidable. Reactivación potenciodinámica electroquímica. Corrosión intergranular.

\section{Evaluation of sensitization in stainless steel AISI 304}

\begin{abstract}
Austenitic stainless steel, when heat-treated at $550-850{ }^{\circ} \mathrm{C}$, became susceptible to intergranular corrosion in acids. This phenomenon, know as sensitization, it is result from the precipitation of chrome carbides in the grain boundary, making these areas less resistant to corrosion. Two different electrochemical reactivation tests are compared with a destructive test and related to the classification of its respective microstructures. It was established a quantitative methodology to evaluate the degree of sensitization in AISI 304 and also to compare the correspondence of the results with the data of the automatic and portable EPR device for non-destructive field measurement of the degree of sensitization. The used electrochemical techniques were the EPR (Electrochemical Potentiokinetic Reactivation) or single loop test and the PRP (Pasivation Reactivation Potentiokinetic) or double loop test. The destructive test used was boiling, $120 \mathrm{~h}$ ferric sulfate- $50 \%$ sulfuric acid, according to the standard practices ASTM A-262 practices B. The classifications of etch structures were according to the standard practices ASTM A-262 practices B.
\end{abstract}

Keywords Sensitization. Stainless steel. Electrochemical potentiodynamic reactivation. Intergranular corrosion.

\section{INTRODUCCIÓN}

Los aceros inoxidables austeníticos se utilizan en la industria petroquímica por su excelente resistencia a la corrosión y características mecánicas; sin mebargo, se han producido fallas inesperadas en equipos industriales en determinadas condiciones de servicios, siendo una de las causas principales la corrosión intergranular cuando este acero se encuentra en estado sensibilizado y en contacto con soluciones corrosivas ${ }^{[1]}$.
La sensibilización de los aceros inoxidables ocurre cuando se exponen a temperaturas entre 500 y $800{ }^{\circ} \mathrm{C}$ durante un tiempo determinado, produciendo la precipitación de carburos de cromo hacia los límites de grano, con lo cual se reduce la concentración de cromo en sus cercanías, haciendo esa área susceptible a la corrosión ${ }^{[2]}$.

Se dispone de varias pruebas para evaluar el grado de sensibilización, algunas de ellas estandarizadas por la norma ASTM A-262. Cuatro de esas prácticas (B-E) corresponden a una exposición

(*) ECOPETROL - Instituto Colombiano del Petróleo. Km. 7, Vía Piedecuesta, Santander, Colombia. 
prolongada del acero a una solución ácida en ebullición, determinando el grado de sensibilización por pérdida de peso, siendo tediosas y destructivas. La prueba ASTM A-262A, consistente en un ataque con ácido oxálico seguido por una evaluación metalográfica de la estructura atacada, es fácil de realizar pero tiene la desventaja de dar resultados cualitativos (prueba no destructiva).

Se dispone de técnicas electroquímicas para medir el grado de sensibilización. Las pruebas de reactivación electroquímica se usan frecuentemente debido a la cualidad de detectar bajos grados de sensibilización, que no pueden ser detectados por otros métodos, permitiendo obtener una medida cuantitativa, fácil y rápida de realizar. Estas pruebas consisten en la exposición de la superficie a evaluar a una solución de ácido sulfúrico diluido, con tiocianato de potasio como activador o pasivador. La muestra se polariza anódicamente hasta el rango pasivo, donde luego se restituye el potencial catódicamente hasta el potencial de corrosión. El comportamiento de disolución del material durante el retorno del potencial (reactivación) se mide para determinar el grado de sensibilización ${ }^{[3]}$.

Este estudio comprende la comparación de dos pruebas de reactivación electroquímica (reactivación potenciocinética electroquímica o simple curva y reactivación potenciocinética de pasivación o doble curva) con el ataque con ácido oxálico (ASTM A-262A) y con la prueba ácida en ebullición de sulfato férrico-ácido sulfúrico (ASTM A262B), en un acero inoxidable AISI 304 después de varios tratamientos térmicos. El objetivo de esta comparación es proponer una base para la selección del método más adecuado y apropiado para determinar el grado de sensibilización y, así mismo, clarificar las características de los métodos electroquímicos y definir las correlaciones de estas pruebas.

El DOS Tester es una herramienta automática y portátil para medir el grado de sensibilización en campo. Se determinaron las ventajas y desventajas para detectar el grado de sensibilización de un acero inoxidable AISI 304 por esta herramienta y su correspondencia con los métodos anteriormente descritos.

\section{METODOLOGÍA EXPERIMENTAL}

\subsection{Material de prueba}

El material usado para esta investigación fue un acero inoxidable austenitico AISI 304 cuya com- posición química se presenta en la tabla I. Las muestras fueron tomadas de una misma barra, cortadas de forma cilíndrica, con un diámetro de $3 \mathrm{~cm}$ y espesor de $0,5 \mathrm{~cm}$. Para partir de una microestructura homogénea en todas las probetas se realizó un tratamiento de solubilización, calentando las probetas a $1.050^{\circ} \mathrm{C}$ durante $18 \mathrm{~h}$, seguido de un temple en agua ${ }^{[4]}$.

Las diferentes variaciones en el grado de sensibilización de las muestras se lograron con un tratamiento de sensibilización a $670{ }^{\circ} \mathrm{C}$, temperatura de máxima sensibilización del acero inoxidable ${ }^{[5]}$, para tiempos de exposición de 0,2, 0,4, 0,7,1,2,3, $4,5,6,7,30,50,70 \mathrm{~h}$ y posteriormente templadas en agua.

\subsection{Pruebas realizadas}

\subsubsection{Pruebas no destructivas}

\subsubsection{Pruebas electroquímicas}

Las pruebas electroquímicas se realizaron en una celda plana, mostrada en la figura 1 , usando como electrolito una solución de $0,5 \mathrm{M}$ de $\mathrm{H}_{2} \mathrm{SO}_{4}+0,01$ $\mathrm{M}$ de $\mathrm{KSCN}$, controlando la temperatura de prueba a $30^{\circ} \mathrm{C}$ por medio de una cinta de calentamiento. Las muestras fueron preparadas con $\mathrm{SiC}$ a $1 \mu$, limpiadas y desengrasadas antes de las pruebas para evitar efectos de borde y poder efectuar análisis metalográficos. El equipo utilizado para efectuar las pruebas electroquímicas de corriente directa fue un Potenciostato/Galvanostato 273 A de E\&G Princenton.

Se realizaron dos pruebas electroquímicas:

- Prueba de simple curva: Se realiza inmersión de la muestra en la solución de prueba hasta alcanzar el potencial de corrosión, el cual es cercano a $-0,45 \mathrm{~V}$ vs SCE. Posteriormente se pasiva la superficie a un potencial de $+200 \mathrm{mV}$ vs SCE manteniéndola durante 2 min y luego el potencial se reversa a una velocidad de $6 \mathrm{~V} / \mathrm{h}$ hasta el potencial de corrosión. El criterio usado para diferenciar las muestras sensibilizadas,

Tabla I. Composición química (\% wt) del acero AISI 304

Table I. Composition of AISI 304 stainless steel (\% wt)

\begin{tabular}{ccccccc}
\hline C & Si & Mn & P & S & Cr & Ni \\
\hline 0,052 & 0,55 & 1,23 & 0,017 & 0,011 & 18,7 & 9,5 \\
\hline
\end{tabular}




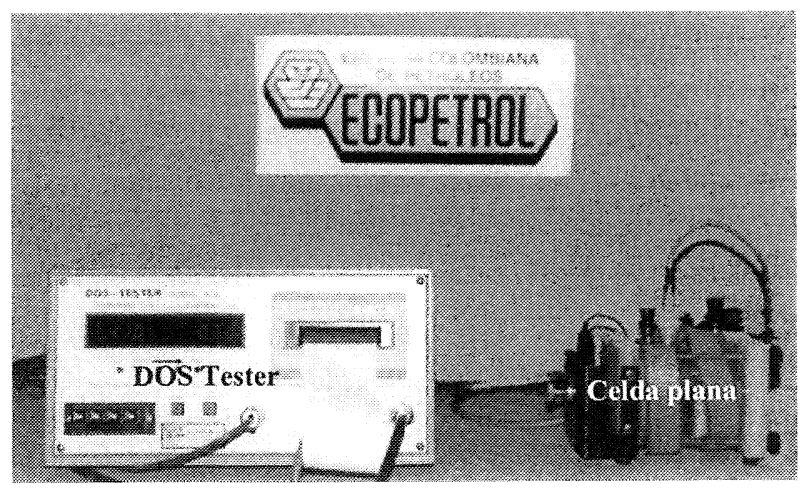

Figura 1. Celda plana utilizada para las pruebas electroquímicas con el DOS Tester y el Potenciostato/ Galvanostato 273 A de E\&G Princenton.

Figure 1. Flat cell used for electrochemical test with DOS Tester and potenciostat/galvanostat 273 A EG \& G.

es la carga de sensibilización (Pa) calculada por la integral de la curva E vs I en el pico de reactivación.

- Prueba de doble curva: Cuando la probeta alcanza el potencial de corrosión, se incrementa el potencial a $+0,3 \mathrm{~V}$ vs SCE a una velocidad de barrido de $100 \mathrm{mV} / \mathrm{min}$; en este potencial se regresa a su potencial de corrosión a la misma velocidad de barrido. El resultado de esta prueba muestra dos curvas en la gráfica E vs I. Las corrientes de máximas de cada curva son medidas, Ia: para la curva anódica e Ir: para la curva de reactivación. La relación Ir/la es usada como una medida del grado de sensibilización.

\subsubsection{Pruebas de ataque con ácido oxálico} (ASTM A-262A)

Se realizó un ataque anódico sobre la superficie de la muestra, previamente pulida a $1 \mu$, con una solución de $10 \%$ ácido oxálico, el fin del procedimiento es producir un ataque que revele una de las siguientes estructuras:

- Step: escalones entre granos.

- Dual: canales entre los límites de grano, sin ser completamente rodeados.

- Ditch: los granos son completamente rodeados por canales.

\subsubsection{Pruebas con el DOS Tester}

Se usó la misma celda, solución, acabado superficial y temperatura de las pruebas electroquímicas descritas anteriormente. Este equipo (Fig. 1) usa la técnica de doble curva, siendo su respuesta la de- terminación automática de la relación de corrientes Ir:Ia.

\subsubsection{Prueba destructiva}

\subsubsection{Prueba de inmersión (ASTM A-262B)}

Se realizó la inmersión de la muestra en una solución en ebullición al 50 \% en peso de ácido sulfúrico más sulfato férrico, durante 120 h. Las muestras son pesadas antes y después de la exposición para determinar la perdida de peso y calcular la velocidad de corrosión en mpy. Se utilizaron 3 Erlenmeyer, cada uno con 4 muestras, incorporando condensadores para evitar perdidas de solución y, por consiguiente, variación en la concentración.

\section{RESULTADOS}

\subsection{Pruebas de simple curva}

La figura 2 presenta las curvas electroquímicas para la prueba de simple curva a diferentes tiempos de exposición a $650^{\circ} \mathrm{C}$, con su respectiva microestructura.

Existe una correspondencia entre la microestructura observada y la carga de activación (área bajo la curva). Para tiempos de exposición cortos $(0,2 \mathrm{~h})$ se observa un bajo grado de sensibilización (estructura Dual) teniendo la carga de activación más baja. Para 50 h de exposición el grado de sensibilización es severo (estructura Ditch) presentando la más alta carga de activación.

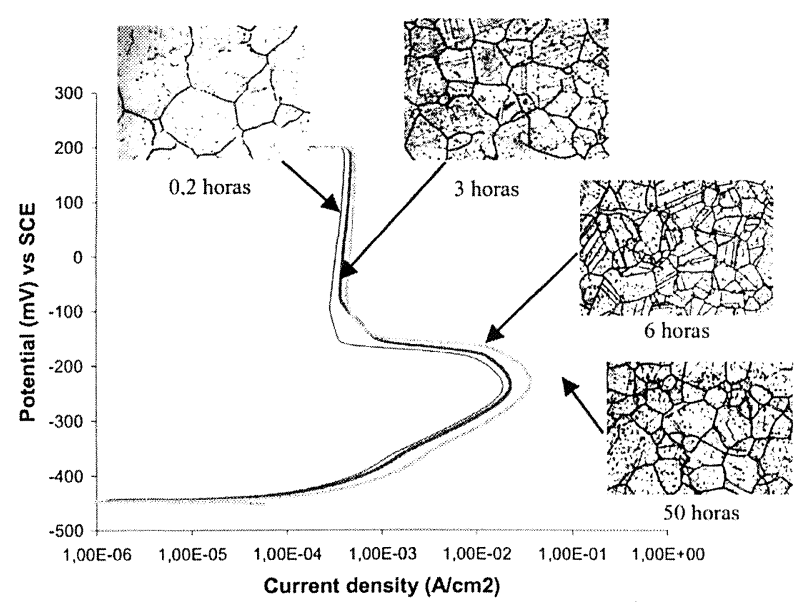

Figura 2. Curvas de polarización para la prueba de simple curva a diferentes tiempos de exposición a $650{ }^{\circ} \mathrm{C}$ en el acero AISI 304. Microestructuras a $100 \mathrm{X}$.

Figure 2. Single loop polarization curves for type 304 stainless steel heated at $650^{\circ} \mathrm{C}$. Microstructures 100X. 
Para el cálculo del parámetro cuantitativo, $\mathrm{Pa}$, se asume que solamente los límites de grano son atacados y que el ancho de ataque es constante. Sin embargo, en las microestructuras se observa que existe variación en el ancho de los límites de grano y que se presenta picado dentro de los granos (causado por inclusiones no metálicas). Estos factores contribuyen adiciones en la carga medida y, por lo tanto, el valor calculado por esta técnica es poco fiable para medir el grado de sensibilización.

\subsection{Pruebas de doble curva}

La figura 3 presenta las curvas electroquímicas para las pruebas de doble curva para diferentes tiempos de exposición a $650^{\circ} \mathrm{C}$, con su respectiva microestructura. Cuanto mayor sea el tiempo de exposición a la temperatura de prueba, mayor será el incremento de la corriente de reactivación, siendo provocado por un mayor grado de sensibilización y por la consiguiente relación de corrientes (Ir:Ia) menores. Las microestructuras observadas después de la prueba muestran buena correlación con la tendencia de la corriente de reactivación, encontrándose estructuras Dual para tiempos menores de $6 \mathrm{~h}$ y estructuras Ditch para mayores tiempos.

Este método tiene la ventaja de que la relación de corrientes no es afectada por el picado o acabado superficial antes de la prueba, debido a que el barrido anódico actúa como un ataque electroquímico y disuelve las inclusiones no metálicas antes del barrido de reactivación.

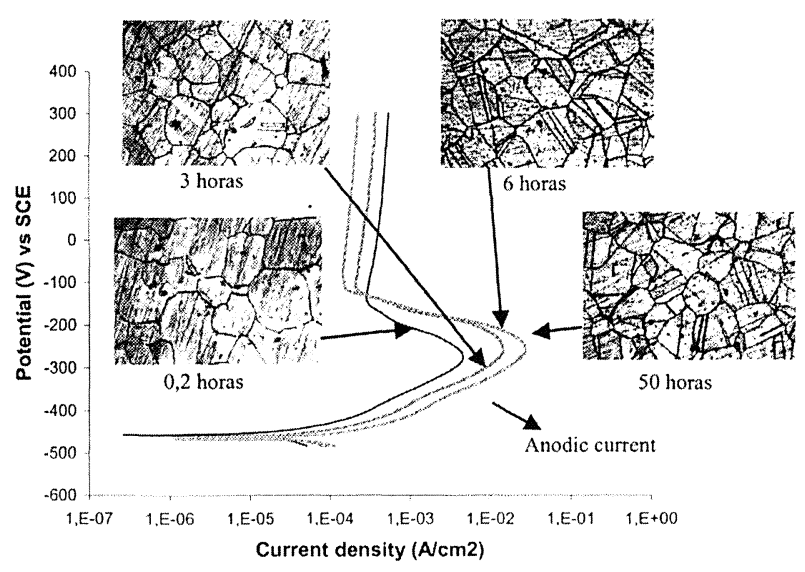

Figura 3. Curvas de polarización para la prueba de doble curva a diferentes tiempos de exposición a $650{ }^{\circ} \mathrm{C}$ en el acero AISI 304. Microestructuras a $100 \mathrm{X}$.

Figure 3. Double loop polarization curves for type 304 stainless steel heated at $650^{\circ} \mathrm{C}$. Microstructures 100X.

\subsection{Prueba con ácido oxálico (ASTM A-262A)}

La figura 4 muestra las microestructuras en el acero AISI 304 después de la prueba con ácido oxálico. Se observa que la longitud de grano atacado incrementa con un aumento del grado de sensibilización de la muestra. Para periodos cortos de exposición a $650^{\circ} \mathrm{C}$, solo una pequeña fracción de los límites de grano es precipitada por carburos de cromo y, por lo tanto, atacado por el oxálico. Para tiempos de exposición más prolongados ocurre la precipitación, también, en los límites de grano menos favorables, de manera que la longitud total de límites de grano atacado se incrementa.

Se observa una correspondencia entre las estructuras reveladas por el ataque con ácido oxálico con las microestructuras después de las pruebas de simple curva y doble curva. Para tiempos de exposición menores a $6 \mathrm{~h}$ se presentan estructuras Dual, donde los límites de grano no están completamente rodeados por el ataque. Para tiempos mayores a $6 \mathrm{~h}$ se presentan estructuras Ditch, donde todos límites de grano son rodeados por la precipitación de carburos y, por consiguiente, socavados por el ataque.

En las maclas y dentro de los granos se aprecia precipitación de carburos de cromo, especialmente después de 6 h de exposición, debido a que se dieron las condiciones favorables de tiempo y temperatura para su precipitación. La prueba de ácido oxálico, aunque no es cuantitativa, es muy usada para diferenciar los materiales que están libres de sensibilización de aquellos que lo presentan.

\subsection{Pruebas con el DOS Tester}

El equipo contiene un microprocesador para medidas completamente automáticas de la prueba de doble curva. Cuando se termina una prueba, los resultados de la relación de corrientes son impresos.

La figura 5 presenta los resultados de la relación de corrientes a diferentes tiempos de exposición utilizando el DOS Tester. Los valores encontrados para estructuras Dual tienen buena correlación con los observados en la prueba de doble curva en el Potenciostato/Galvanostato 273 A de E\&G Princenton. Sin embargo el DOS Tester no tiene la capacidad de detectar altos grados de sensibilización (estructuras Ditch) debido a que las corrientes anódicas sobrepasan los $80 \mathrm{~mA}$, que es el valor límite de corrientes manejadas por el DOS Tester.

Los equipos portátiles electroquímicos de campo tienen la ventaja de poder fijar los parámetros 


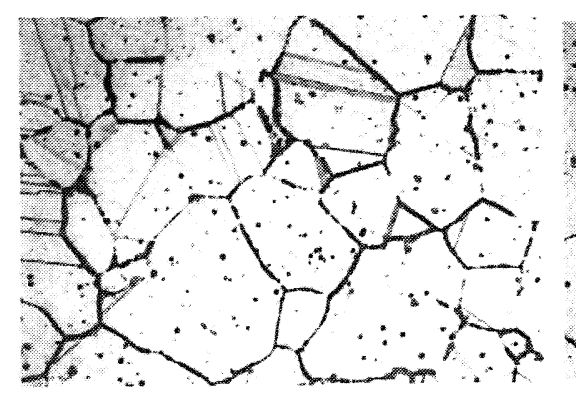

a)

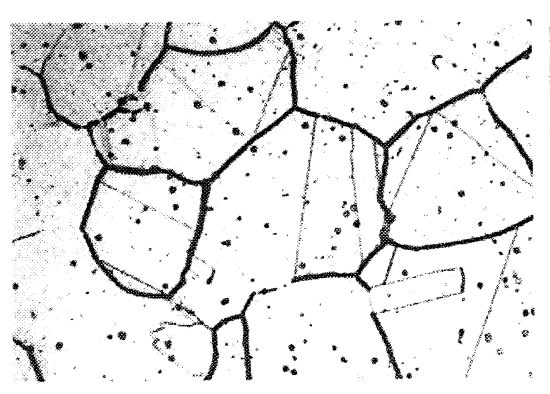

b)

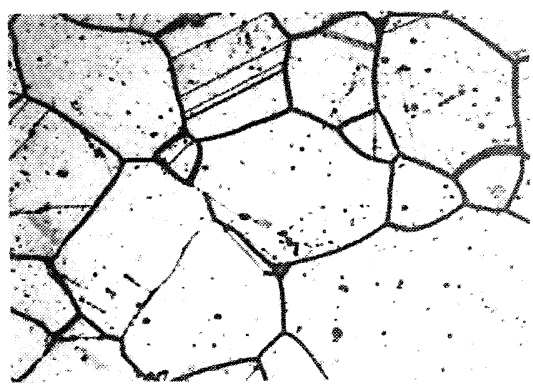

c)

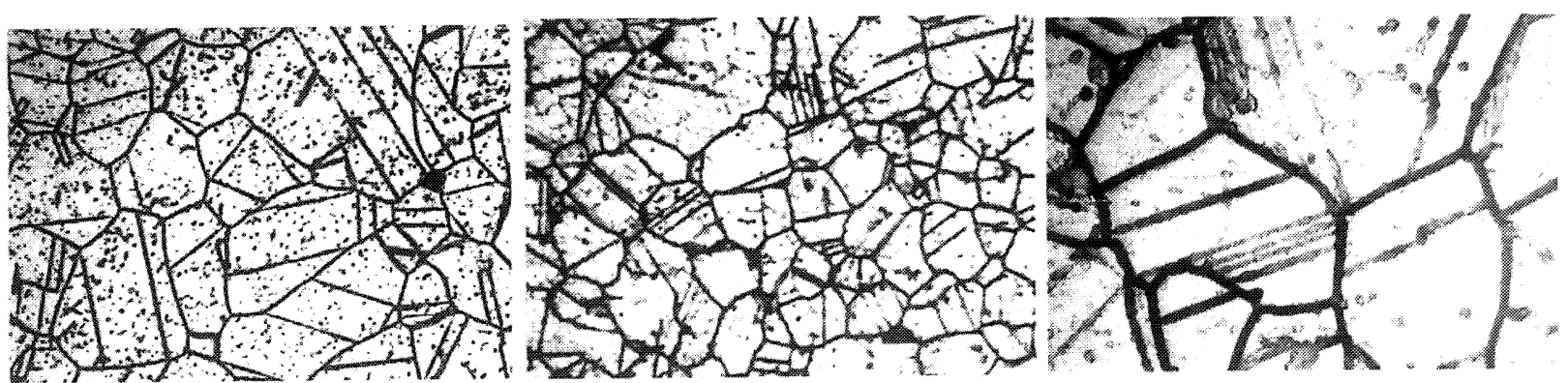

d)

e)

1)

Figura 4. Estructura atacada después de la prueba de ácido oxálico: a) 0,2 h. (100X), b) 1 h. (100X), c) 3 h. (100X), d) 6 h. $(100 X)$, e) 50 h. (100X), f) 50 h. (500X).

Figure 4. Etch structure after the oxalic acid test heated at $650{ }^{\circ} \mathrm{C}$ : a) $\left.\left.0.2 \mathrm{~h} .(100 \mathrm{X}), \mathrm{b}\right) 1 \mathrm{~h} .(100 \mathrm{X}), \mathrm{c}\right) 3 \mathrm{~h}$. (100X), d) $6 \mathrm{~h}$. $(100 X)$, e) 50 h. (100X), f) 50 h. (500X).

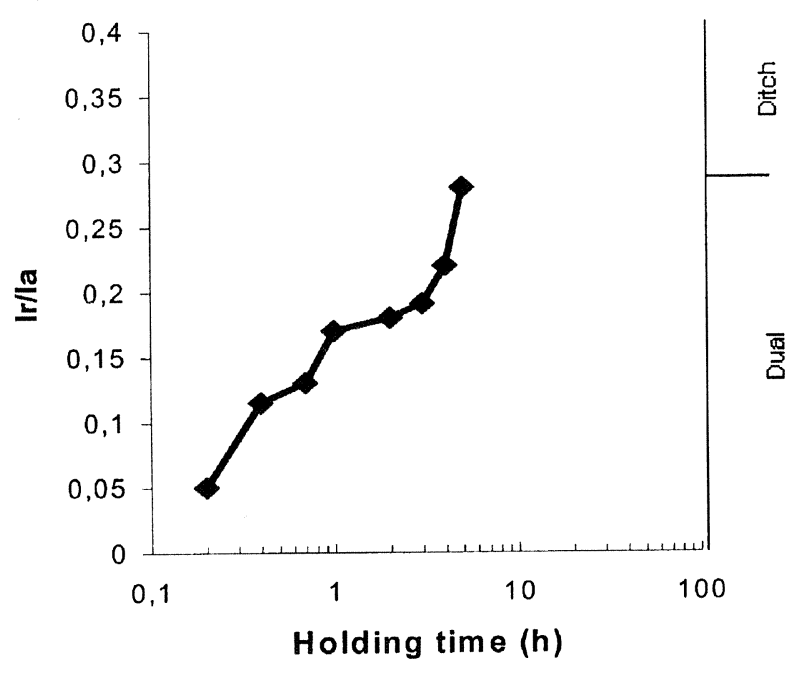

Figura 5. Relación de corrientes del equipo DOS Tester para diferentes tiempos de sensibilización.

Figure 5. Current ratio of DOS tester device for differing sensitization time.

84 de los ensayos, permitiendo realizar pruebas electroquímicas de doble curva, teniendo límites de corriente altos, superando los inconvenientes del DOS Tester. Se realizaron pruebas electroquímicas con el Potentyodyne $4300 \mathrm{~b}$ de doble curva, los valores obtenidos tienen buenas correlaciones con los observados con el Potenciostato/Galvanostato 273 A de E\&G Princenton.

\subsection{Prueba de inmersión en ácido sulfúrico- sulfato férrico}

La figura 6 muestra el efecto del tiempo de sensibilización a $650^{\circ} \mathrm{C}$ del acero AISI 304 en la velocidad de corrosión para la prueba de ácido sulfúrico-sulfato férrico (ASTM A-262 B), además presenta la comparación con la prueba de doble curva y ácido oxálico (ASTM A-262 A).

Rev. Metal. Madrid Vol. Extr. (2003) 80-85 


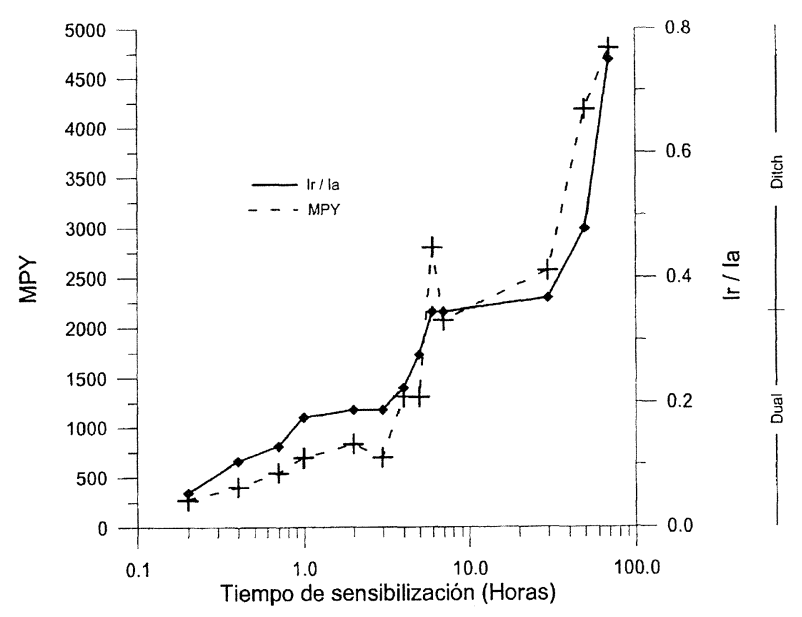

Figura 6. Relación entre la prueba de doble curva, la prueba de sulfato férrico-ácido sulfúrico (ASTM A-262B) y la prueba de ataque con ácido oxálico (ASTM A-262A) para diferentes grados de sensibilización.

Figure 6. Relationship between reactivation ratio (double loop test), ferric sulfate-sulfuric acid test (ASTM A-262B) and oxalic etch test (ASTM A-262A) for differing sensitization grade.

La velocidad de corrosión en esta prueba es calculada por la pérdida de peso, que es una medida del número de límites de grano que han sido socavados y granos arrancados. Por lo tanto, a mayor grado de sensibilización mayor número de granos pueden ser más fácilmente atacados, incrementando la velocidad de corrosión. Las estructuras Dual, donde los límites de grano son parcialmente atacados, se tienen velocidades de corrosión más bajas que las estructuras Ditch, donde todos los límites de grano son atacados.

Existe una buena relación entre las pruebas de inmersión en ácido sulfúrico-sulfato férrico y la prueba electroquímica de doble curva, presentán- dose una diferencia clara en el comportamiento de las curvas para estructuras Dual y Dich. Una vez formado la estructura Ditch, para $6 \mathrm{~h}$ de exposición, la velocidad de corrosión se acelera notoriamente, siendo este punto el valor crítico del grado de sensibilización a las condiciones de la prueba de inmersión, correspondientes a un valor de 0,34 para la relación de corrientes en la prueba de doble curva.

\section{CONCLUSIONES}

Los resultados de la prueba de doble curva muestran una buena correlación con la prueba de inmersión especificada por la norma ATM A-262 B, permitiendo comparar los resultados cuantificados por la prueba de doble curva con resultados de corrosión intergranular sobre materiales con similar grado de sensibilización en un medio dado.

La prueba de doble curva tiene la capacidad de detectar cuantitativamente desde muy bajos, hasta altos grados de sensibilización; siendo el método más conveniente para monitorear los cambios de sensibilización en materiales que presenten susceptibilidad por corrosión intergranular.

\section{REFERENCIAS}

[1] F. Umemura, Y. Hanai y T. KaWamoto, IHI Eng. Rev. 162 (1982) $1-7$

[2] R. Jargelius, S. Hertzman, E. Symniotis, H. Hannien y P. Aaltonen, Corrosion 476 (1991) 429-435.

[3] W. Clark, V.M. Romero y J.C. Danko, Corrosion Paper 180 (1977).

[4] J.H. Payer y R.W. Staehle, Corrosion 311 (1975) 31-35.

[5] Mignone, A. Borrello y A. la Barbera, Corrosion 387 (1982) 390-402. 